\title{
The temporal paradox of Hebbian learning and homeostatic plasticity
}

\author{
Friedemann Zenke ${ }^{\mathrm{a}, *}$, Wulfram Gerstner ${ }^{\mathrm{b}}$, Surya Ganguli ${ }^{\mathrm{a}}$ \\ ${ }^{a}$ Department of Applied Physics, Stanford University, Stanford, CA 94305, USA \\ ${ }^{b}$ Brain Mind Institute, School of Life Sciences and School of Computer and Communication Sciences, Ecole Polytechnique \\ Fédérale de Lausanne, CH-1015 Lausanne EPFL, Switzerland
}

\begin{abstract}
Hebbian plasticity, a synaptic mechanism which detects and amplifies co-activity between neurons, is considered a key ingredient underlying learning and memory in the brain. However, Hebbian plasticity alone is unstable, leading to runaway neuronal activity, and therefore requires stabilization by additional compensatory processes. Traditionally, a diversity of homeostatic plasticity phenomena found in neural circuits are thought to play this role. However, recent modelling work suggests that the slow evolution of homeostatic plasticity, as observed in experiments, is insufficient to prevent instabilities originating from Hebbian plasticity. To remedy this situation, we suggest that homeostatic plasticity is complemented by additional rapid compensatory processes, which rapidly stabilize neuronal activity on short timescales.

Keywords: synaptic plasticity, Hebbian plasticity, homeostatic plasticity, rapid compensatory processes
\end{abstract}

\section{Introduction}

More than half a century ago, Donald Hebb [1] laid down an enticing framework for the neurobiological basis of learning, which can be succinctly summarized in the well-known mantra, "neurons that fire together wire together" [2]. However, such dynamics suffers from two inherent problems. First, Hebbian learning exhibits a positive feedback instability: those neurons that wire together will fire together more, leading to even stronger connectivity. Second, such dynamics alone would lead to all neurons in a recurrent circuit wiring together, precluding the possibility of rich patterns of variation in synaptic strength that can encode, through learning, the rich structure of experience. Two fundamental ingredients required to solve these problems are stabilization [3], which prevents runaway neural activity, and competition [4-6], in which the strengthening of a synapse may come at the expense of the weakening of others.

In theoretical models, competition and stability are often achieved by augmenting Hebbian plasticity with additional constraints $[3,5,7]$. Such constraints are typically implemented by imposing upper limits on individual synaptic strengths, and by enforcing some constraint on biophysical variables, for example, the

\footnotetext{
${ }^{*}$ Corresponding author

Email addresses: fzenke@stanford.edu (Friedemann Zenke), wulfram.gerstner@epfl.ch (Wulfram Gerstner), sganguli@stanford.edu (Surya Ganguli)
} 
total synaptic strength or average neuronal activity [6-12]. In neurobiology, forms of plasticity exist which seemingly enforce such limits or constraints through synaptic scaling in response to firing rate perturbations $[13,14]$, or through stabilizing adjustments of the properties of plasticity in response to the recent synaptic history, a phenomenon known as homeostatic metaplasticity $[6,11,15,16]$. Overall, synaptic scaling and metaplasticity, as special cases of homeostatic mechanisms that operate over diverse spatiotemporal scales across neurobiology [17-22], are considered key ingredients that contribute both stability and competition to Hebbian plasticity by directly affecting the fate of synaptic strength.

The defining characteristic of homeostatic plasticity is that it drives synaptic strengths so as to ensure a homeostatic set point [23, 24], such as a specific neuronal firing rate or membrane potential. However, it is important that this constraint is implemented only on average, over long timescales, thereby allowing neuronal activity to fluctuate on shorter timescales, so that these neuronal activity fluctuations, which drive learning through Hebbian plasticity, can indeed reflect the structure of ongoing experience. This requisite separation of timescales is indeed observed experimentally; forms of Hebbian plasticity can be induced on the timescale of seconds to minutes [25-28], whilst most forms of homeostatic synaptic plasticity operate over hours or days $[14,24,29]$. This separation of timescales, however, raises a temporal paradox: homeostatic plasticity then may become too slow to stabilize the fast positive feedback instability of Hebbian learning. Indeed modeling studies that have attempted to use homeostatic plasticity mechanisms to stabilize Hebbian learning [11, 30-34] were typically required to speed up homeostatic plasticity to timescales that are orders of magnitude faster than those observed in experiments (Fig. 1).

This temporal paradox could have two potential resolutions. First, the timescale of Hebbian plasticity, as captured by recent plasticity models fit directly to data from slice experiments [28, 35-38], may overestimate the rate of plasticity that actually occurs in-vivo. This overestimate could arise from differences in slice and in-vivo preps, or because complex nonlinear synaptic dynamics, both present in biological synapses and useful in learning and memory [39-41], are missing in most, but not all [42-44], data-driven models. While slow plasticity may be a realistic possibility in cortical areas exhibiting plastic changes over days [45, 46], it may not be a realistic resolution in other areas, like the hippocampus, which must rapidly encode new episodic information $[47,48]$. The second potential resolution to the paradoxical separation of timescales between Hebbian and homeostatic plasticity may be the existence of as yet unidentified rapid compensatory processes (RCPs) that stabilize Hebbian learning. Below, we explore both the theoretical utility and potential neurobiological instantiations of these putative RCPs.

\section{The temporal paradox of Hebbian and homeostatic plasticity}

To understand the theoretical necessity for RCPs to stabilize Hebbian plasticity, it is useful to view a diversity of synaptic learning models through the unifying lens of control theory (Fig. 2a). Here we can 
view the "fire together, wire together" interplay of neuronal activity and Hebbian synaptic plasticity as an unstable dynamical system. Also, we can view a compensatory process as a feedback controller that observes some aspect of either neuronal activity or synaptic strength, and uses this observation to compute a feedback control signal which then directly affects synaptic strength so as to stabilize global circuit activity. Indeed homeostatic plasticity is often thought of as a negative feedback control process [23, 24, 64, 65]. In general, the delay in any feedback control loop must be fast relative to the time-scale over which the unstable system exhibits run-away activity [66]. If the loop is slightly slow, the run-away will start before the stabilizing feedback arrives, generating oscillations within the system. In some cases, if the loop is even slower, the unstable runaway process might escape before stabilization is even possible (Fig. 2b).

Such oscillations and run-away are demonstrated in Fig. 3 for several compensatory mechanisms with feedback timescales that are chosen to be too slow, including the Bienenstock-Cooper-Munro (BCM) rule [6, 67], and triplet spike-timing-dependent plasticity (STDP) [36] with either synaptic scaling [13, 53] or a metaplastic sliding threshold, as stabilizing controllers. For example, the BCM rule (Fig. 3a) can be thought of as a feedback control system where the controller observes a recent average of the postsynaptic output firing rate of a neuron, and uses this information to control both the sign and amplitude of associative plasticity; if the recent average is high (low), plasticity is modulated to be anti-Hebbian (Hebbian). However, to achieve stability, the BCM controller must average recent output-activity over a short enough time-scale to modulate plasticity before this activity itself runs away (Fig. 3a; [11, 31, 32]). This result is not limited to BCM like rate models, but applies equally to STDP models which rely on similar metaplasticity processes to ensure stability ( Fig. 3bc; [31, 36, 37, 52]). These modified STDP rules can be thought of as employing feedback controllers which also observe a recent average of output firing rate, but use this information to modulate the STDP window, thereby stabilizing the system by changing relative rates of long-term potentiation (LTP) and long-term depression (LTD).

Another class of models relies on re-normalization of afferent synaptic weights to stabilize Hebbian plasticity. We distinguish between models with instantaneous algorithmic re-normalization of the weights $[4,5,7,50]$ and models which proportionally scale synaptic weights in an activity dependent way $[8,31,68]$. In the former case the controller observes total synaptic strength and uses this information to adjust synapses to keep this total strength constant. In the latter case, the controller observes neuronal firing rate, or recent average thereof, and uses this information to proportionally adjust afferent synaptic weights to enforce either a specified target output, or a total synaptic strength. Just as in the case of metaplasticity discussed above, the temporal average of the activity sensor has to be computed over a short timescale, related to the timescale over synaptic strengths and neuronal activity change, to ensure stability (Fig. 3d [31-33]). Moreover, the rate at which the synaptic scaling process itself causes synaptic strengths to scale must be finely tuned to a narrow parameter regime that is neither too fast nor too slow [31-33, 53]; if too slow, then stabilization is 
not possible, while if too fast, the stabilization process overshoots, causing oscillations.

Finally, some STDP models can be intrinsically stable, especially in feedforward circuits. One example are pair-based STDP models in which the integral of the STDP window is slightly biased toward depression. When weights are additionally limited by hard bounds, this can lead to bimodal weight distributions and firing rate stabilization $[9,69]$. However, these learning rules typically require fine-tuning and become unstable when input correlations are non-negligible. Other stable models arise from a weight dependence in the learning rule such that high (low) synaptic strength makes LTP (LTD) weaker [53, 70-74]. Such a stabilizing weight dependence is advantageous because it can lead to more plausible unimodal weight distributions as observed empirically $[72,74]$. However, the unimodal weight distribution, by precluding multi-stability in the configurations of synaptic strengths, typically leads to the rapid erasure of synaptic memory traces in the presence of background activity driving plasticity [73, 75]. Moreover, when such weight dependent synaptic learning rules are embedded in a recurrent neuronal circuit without any additional control mechanisms, they can succumb to runaway neural activity as experience dependent neural correlations emerge in the recurrent circuit $[73,76,77]$.

In summary, empirical findings and control-theoretic considerations suggest that compensatory mechanisms capable of stabilizing Hebbian plasticity must operate on tightly constrained timescales. In practice, such compensatory mechanisms must act on similar or even faster timescales than Hebbian plasticity itself $[31,32,36,37,50,52,68]$. STDP, as one of the most common manifestations of Hebbian plasticity in the brain, can be induced in a matter of seconds to minutes [25-27, 78, 79]. Homeostatic plasticity, on the other hand, acts on much longer timescales of hours to days $[14,16,19,29,55,56,80]$. This separation of timescales poses a temporal paradox as it renders most data-driven STDP models unstable [3, 81]. In spiking network models, this instability has severe consequences (Fig. 3b-d); it precludes the emergence of stable synaptic structures or memory engrams [31, 73, 76], unless the underlying plasticity models are augmented by RCPs $[31,50,51,68,82]$. Overall, these considerations suggest that one or more RCPs exist in neurobiological systems, which are missing in current plasticity models.

\section{Putative rapid compensatory processes}

What putative RCPs could augment Hebbian plasticity with the requisite stability and competition? Here we focus on several possibilities, operating at either the network, the neuronal or the dendritic level (Fig. 4a). However, we note that these possibilities are by no means exhaustive.

At the network level, recurrent or feedforward synaptic inhibition could influence and potentially stabilize plasticity at excitatory synapses directly. For instance, [83] demonstrated via dynamic-clamp that a simulated increase in total excitatory and inhibitory background conductance, which could originate from elevated levels of network activity, rapidly reduces the amplitude of LTP, but not LTD, of the STDP window. 
This rapid effect may be mediated through changes in calcium dynamics in dendritic spines and could constitute an RCP. Another study [84] showed that global inhibition in a rate-based network model is sufficient to stabilize plasticity at excitatory synapses with a sliding presynaptic and fixed postsynaptic plasticity threshold. Finally, using a model-based approach, Wilmes et al. [85] have proposed that dendritic inhibition could exert binary switch-like control over plasticity by gating back-propagating action potentials.

Other modeling studies have suggested a role for inhibitory synaptic plasticity (ISP) [86, 87], instead of non-plastic inhibition, in stabilizing Hebbian plasticity. It has been suggested, for instance, that ISP in conjunction with a fixed plasticity threshold at the excitatory synapse could have a similar effect as the sliding threshold in the BCM model [88]. Finally, in some experiments excitatory and inhibitory plasticity are not integrated as independent events, but can influence each other. For instance, there are cases in which induction of ISP alone can flip the sign of subsequent plasticity at excitatory synapses [89]. While inhibition and ISP may act as RCPs, a clear picture of how these elements tie together has not yet emerged. Further experimental and theoretical work is required to understand their potential for acting as RCPs.

Neuromodulation may also play an important role in stabilizing plasticity. Neuromodulators have been implicated in both homeostatic signalling [21] and in gating the expression of synaptic plasticity [90-93]. However, to successfully serve as RCP, neuromodulatory mechanisms have to either drive rapid compensatory changes directly, or substantially reduce the average rate of Hebbian plasticity in-vivo to enable slower forms of homeostatic plasticity to preserve stability. While a detailed account of the role of neuromodulators goes beyond the scope of this article (but see [93, 94] for reviews), here we note that several neuromodulators at least partially meet one of these core requirements for RCPs. For example, nitric oxide (NO), which is involved in synaptic homeostasis and plasticity [95], is released in response to increased NMDA activity and decays within some tens of seconds [96]. Because cell membranes are permeable to NO, the molecule diffuses rapidly and thus could potentially act as a fast proxy of bulk neuronal activity that can be read out locally [97]. Similarly, dopaminergic transmission can be fast [98] and is known to affect induction, consolidation and possibly maintenance of synaptic long-term plasticity [91, 93, 99-101]. A sensible gating strategy implemented by dopamine or other neuromodulators could result in a drastically reduced and more manageable average rate of plasticity in vivo. In contrast, in vitro such neuromodulatory mechanisms might be disengaged, thus potentially creating less natural and much faster plasticity rates compared to in vivo. Apart from the neuromodulatory system, recent work has highlighted the complexity of the local interactions between astrocytes and synaptic plasticity which may also act as RCPs [102-104]. However, to unequivocally answer which aspects of neuromodulation and glial interactions constitute suitable RCPs will require further experimental and theoretical work.

A more well studied possibility for an RCP is heterosynaptic plasticity, which operates at the level of individual neurons or potentially dendritic branches. Heterosynaptic plasticity refers to a non input-specific 
change at other synapses onto a neuron that are not directly activated ([30, 105-108]). Its viability as putative RCP arises from the fact that some forms of heterosynaptic plasticity can be induced rapidly, and moreover, similar to synaptic scaling, can show aspects of weight normalization [106]. For instance, a rapid form of heterosynaptic plasticity, in which neuronal bursting causes bi-directional weight-dependent changes in afferent synapses, has been observed recently $[107,109]$. While the observed weight-dependence is reminiscent of Oja's rule [8], as strong synapses weaken, it is not identical because weak synapses can also strengthen. Nevertheless, this form of heterosynaptic plasticity has been shown to prevent runaway of LTP in models of feedforward circuits [30] and has been demonstrated to co-occur with Hebbian plasticity in experiments [109].

Recently, the utility in stabilizing runaway LTP has also been demonstrated in a recurrent network model of spiking neurons [82], in which a similarly burst dependent form of heterosynaptic plasticity is crucial to ensure stable formation and recall of Hebbian cell assemblies (Fig. 4b). Moreover, the work suggests a potential role of heterosynaptic plasticity in triggering the reversal of LTP and LTD [110].

At the level of dendritic branches, a recently described form of structural heterosynaptic plasticity [108], involves local dendritic competition between synapses. Specifically, glutamate induced structural synaptic potentiation of a set of clustered dendritic spines causes shrinkage of nearby, but unstimulated spines. Interestingly, even when structural potentiation was switched off through inhibition of $\mathrm{Ca}^{2+} /$ calmodulindependent protein kinase II (CaMKII), the heterosynaptic effect persists, suggesting a model in which spines send and receive shrinkage signals instead of competing for limited resources. Moreover, the observed dendritic locality is consistent with work on local or branch specific conservation of total synaptic conductance $[106,111]$.

Finally, in some cases heterosynaptic plasticity might not actually be heterosynaptic, as it may still depend on low levels of spontaneous synaptic activity in unstimulated synapses [112]. A recent biophysical model derived from synaptic plasticity data [113] actually requires such low-levels of activity to match the data. Interestingly, this model suggests such "heterosynaptic" plasticity could arise as a consequence of a rapid (timescale $\sim 12 \mathrm{~s}$ ) homeostatic sliding threshold possibly related to autophosphorylation of CaMKII.

While heterosynaptic plasticity, like synaptic scaling, can have a stabilizing effect, it is distinct from synaptic scaling in two ways. First, heterosynaptic plasticity need not multiplicatively scale all weights in the same manner. Second, it is unclear whether heterosynaptic plasticity in general drives neuronal activity variables to a specific set point, like synaptic scaling does [24]. Thus, while heterosynaptic plasticity has the rapidity to act as a putative $\mathrm{RCP}$, its functional utility in storing memories requires further empirical and theoretical study, as it may lack the ability to precisely preserve ratios of synaptic strengths. However, its clear functional utility in preventing instability (Fig. 3d), along with even an approximate preservation in ratios of strengths, could potentially endow the interaction of heterosynaptic plasticity and Hebbian learning 
with the ability to stably learn and remember memories. Further network modeling, building on promising heterosynaptic plasticity models [30, 82, 113], could be highly instructive in elucidating the precise properties, beyond rapidity, a putative RCP must obey in order to provide appropriate competition and stability to Hebbian plasticity.

\section{Conclusion}

The trinity of Hebbian plasticity, competition and stability are presumed to be crucial for effective learning and memory. However, a detailed theoretical and empirical understanding of how these diverse elements conspire to functionally shape neurobiological circuits is still missing. Here we have focused on one striking difference between existing models and neurobiology: the paradoxical separation of timescales between Hebbian and homeostatic plasticity. In models, such a separation of timescales typically leads to instability, unless plasticity is constrained by RCPs that act much faster than observed forms of homeostatic plasticity. In principle, RCPs could be implemented at various spatial scales. Here we have primarily discussed different forms of heterosynaptic plasticity and processes involving synaptic inhibition as possible candidates. However, rapid processes involving neuromodulation, glial interactions, or intrinsic plasticity [114-117] could also constitute RCPs. Thus, identifying the key neurobiological processes that provide stability and competition to Hebbian learning rules remains an important direction for future research.

\section{Acknowledgements}

FZ was supported by the SNSF (Swiss National Science Foundation). SG was supported by the Burroughs Wellcome, Sloan, McKnight, Simons and James S. McDonnell foundations and the Office of Naval Research. WG was supported for this work by the European Research Council under grant agreement number 268689 (MultiRules) and by the European Community's Seventh Framework Program under grant no. 604102 (Human Brain Project).

[1] D. O. Hebb, The Organization of Behavior: A Neuropsychological Theory, Wiley \& Sons New York, 1949.

[2] C. J. Shatz, The Developing Brain, Scientific American 267 (3) (1992) 60-67, URL http://www. scientificamerican.com/article/the-developing-brain/.

[3] L. F. Abbott, S. B. Nelson, Synaptic plasticity: taming the beast, Nat Neurosci 3 (2000) 1178-1183, ISSN 1097-6256, doi:10.1038/81453, URL http://www.nature.com/neuro/journal/v3/n11s/full/ nn1100_1178.html. 
[4] (•) N. Rochester, J. Holland, L. Haibt, W. Duda, Tests on a cell assembly theory of the action of the brain, using a large digital computer, IEEE Trans Inf Theory 2 (3) (1956) 80-93, ISSN 0096-1000, doi:10.1109/TIT.1956.1056810.

Of historical interest as probably the earliest modeling study - the authors show that Hebbian LTP needs to be complemented by two mechanisms: (i) LTD formulated as an early version of a covariance rule and (ii) and a rapid heterosynaptic mechanism formulated as normalization of the sum over all weights of afferent synapses.

[5] C. von der Malsburg, Self-organization of orientation sensitive cells in the striate cortex, Kybernetik 14 (2) (1973) 85-100, ISSN 0023-5946.

[6] (•) E. Bienenstock, L. Cooper, P. Munro, Theory for the development of neuron selectivity: orientation specificity and binocular interaction in visual cortex, J Neurosci 2 (1) (1982) 32-48, URL http: //www. jneurosci.org/cgi/content/abstract/2/1/32.

This classic modeling paper for metaplasticity via a sliding threshold between LTP and LTD links thorough mathematical analysis to experimental data available at that time.

[7] (•) K. D. Miller, D. J. MacKay, The role of constraints in Hebbian learning, Neural Comput 6 (1) (1994) $100-126$.

A careful and transparent mathematical study on the role of synaptic constraints in Hebbian learning.

[8] E. Oja, Simplified neuron model as a principal component analyzer, J Math Biol 15 (3) (1982) 267-273, ISSN 0303-6812, doi:10.1007/BF00275687, URL http://www.springerlink.com/content/ u9u6120r003825u1/abstract/.

[9] R. Kempter, W. Gerstner, J. van Hemmen, Hebbian learning and spiking neurons, Phys Rev E 59 (4) (1999) 4498-4514, ISSN 1063-651X, 1095-3787, doi:10.1103/PhysRevE.59.4498, URL http: //infoscience.epfl.ch/record/97790.

[10] S. Song, K. D. Miller, L. F. Abbott, Competitive Hebbian learning through spike-timing-dependent synaptic plasticity, Nat Neurosci 3 (9) (2000) 919-926, doi:10.1038/78829, URL http://www.nature . com/neuro/journal/v3/n9/full/nn0900_919.html.

[11] L. N. Cooper, N. Intrator, B. S. Blais, H. Z. Shouval, Theory of Cortical Plasticity, World Scientific, New Jersey, ISBN 978-981-238-746-2, 2004.

[12] C. S. N. Brito, W. Gerstner, Nonlinear Hebbian Learning as a Unifying Principle in Receptive Field Formation, PLOS Comput Biol 12 (9) (2016) e1005070, ISSN 1553-7358, doi:10.1371/journal.pcbi.1005070, URL http://journals.plos.org/ploscompbiol/article?id=10.1371/journal.pcbi.1005070. 
[13] G. G. Turrigiano, K. R. Leslie, N. S. Desai, L. C. Rutherford, S. B. Nelson, Activity-dependent scaling of quantal amplitude in neocortical neurons, Nature 391 (6670) (1998) 892-896, ISSN 0028-0836, doi: 10.1038/36103, URL http://dx.doi.org/10.1038/36103.

[14] G. G. Turrigiano, The Self-Tuning Neuron: Synaptic Scaling of Excitatory Synapses, Cell 135 (3) (2008) 422-435, ISSN 0092-8674, doi:10.1016/j.cell.2008.10.008, URL http://www.sciencedirect. com/science/article/pii/S0092867408012981.

[15] W. C. Abraham, M. F. Bear, Metaplasticity: The plasticity of synaptic plasticity, Trends Neurosci 19 (4) (1996) 126-130, ISSN 0166-2236, doi:10.1016/S0166-2236(96)80018-X, URL http://www . sciencedirect.com/science/article/pii/S016622369680018X.

[16] W. C. Abraham, Metaplasticity: Tuning synapses and networks for plasticity, Nat Rev Neurosci 9 (5) (2008) 387-387, ISSN 1471-003X, doi:10.1038/nrn2356, URL http://www .nature.com/nrn/journal/ v9/n5/abs/nrn2356.html.

[17] G. W. Davis, Homeostatic Control of Neural Activity: From Phenomenology to Molecular Design, Annu Rev Neurosci 29 (1) (2006) 307-323, doi:10.1146/annurev.neuro.28.061604.135751, URL http: //www. annualreviews.org/doi/abs/10.1146/annurev.neuro.28.061604.135751.

[18] E. Marder, J.-M. Goaillard, Variability, compensation and homeostasis in neuron and network function, Nat Rev Neurosci 7 (7) (2006) 563-574, ISSN 1471-003X, doi:10.1038/nrn1949, URL http://www . nature.com/nrn/journal/v7/n7/abs/nrn1949.html.

[19] I. Rabinowitch, I. Segev, Two opposing plasticity mechanisms pulling a single synapse, Trends in Neurosciences 31 (8) (2008) 377-383, ISSN 0166-2236, doi:10.1016/j.tins.2008.05.005, URL http:// www.sciencedirect.com/science/article/pii/S0166223608001483.

[20] G. Turrigiano, Too Many Cooks? Intrinsic and Synaptic Homeostatic Mechanisms in Cortical Circuit Refinement, Annu Rev Neurosci 34 (1) (2011) 89-103, doi:10.1146/annurev-neuro-060909-153238, URL http://www . annualreviews .org/doi/abs/10.1146/annurev-neuro-060909-153238.

[21] G. G. Turrigiano, Homeostatic Synaptic Plasticity: Local and Global Mechanisms for Stabilizing Neuronal Function, Cold Spring Harb Perspect Biol 4 (1) (2012) a005736, ISSN , 1943-0264, doi: 10.1101/cshperspect.a005736, URL http://cshperspectives.cshlp.org/content/4/1/a005736.

[22] J. Gjorgjieva, J. F. Evers, S. J. Eglen, Homeostatic Activity-Dependent Tuning of Recurrent Networks for Robust Propagation of Activity, J. Neurosci. 36 (13) (2016) 3722-3734, ISSN 0270-6474, 1529-2401, doi:10.1523/JNEUROSCI.2511-15.2016, URL http://www . jneurosci.org/content/36/13/3722. 
[23] W. B. Cannon, The wisdom of the body, Norton, New York, ISBN 0-393-00205-5 978-0-393-00205-8, 1967.

[24] (•) G. G. Turrigiano, The dialectic of Hebb and homeostasis, Phil. Trans. R. Soc. B 372 (1715) (2017) 20160258, ISSN 0962-8436, 1471-2970, doi:10.1098/rstb.2016.0258, URL http://rstb. royalsocietypublishing.org/content/372/1715/20160258. A broad review of synaptic homeostatic plasticity which also addresses open questions regarding timescales of plasticity and homeostasis.

[25] H. Markram, J. Lübke, M. Frotscher, B. Sakmann, Regulation of synaptic efficacy by coincidence of postysnaptic AP and EPSP, Science 275 (1997) 213-215.

[26] G.-Q. Bi, M.-M. Poo, Synaptic Modifications in Cultured Hippocampal Neurons: Dependence on Spike Timing, Synaptic Strength, and Postsynaptic Cell Type, J Neurosci 18 (24) (1998) 10464 -10472, URL http://www . jneurosci.org/content/18/24/10464.abstract.

[27] L. I. Zhang, H. W. Tao, C. E. Holt, W. A. Harris, M.-m. Poo, A critical window for cooperation and competition among developing retinotectal synapses, Nature 395 (6697) (1998) 37-44, ISSN 0028-0836, doi:10.1038/25665, URL http://www.nature.com/nature/journal/v395/n6697/ abs/395037a0.html.

[28] R. C. Froemke, Y. Dan, Spike-timing-dependent synaptic modification induced by natural spike trains., Nature 416 (6879) (2002) 433-8, ISSN 0028-0836, doi:10.1038/416433a, URL http://www.ncbi.nlm. nih.gov/pubmed/11919633.

[29] A. J. Watt, N. S. Desai, Homeostatic plasticity and STDP: keeping a neuron's cool in a fluctuating world, Front Synaptic Neurosci 2 (2010) 5, doi:10.3389/fnsyn.2010.00005, URL http://www . frontiersin.org/synaptic_neuroscience/10.3389/fnsyn.2010.00005/abstract.

[30] J.-Y. Chen, P. Lonjers, C. Lee, M. Chistiakova, M. Volgushev, M. Bazhenov, Heterosynaptic Plasticity Prevents Runaway Synaptic Dynamics, J Neurosci 33 (40) (2013) 15915-15929, ISSN 0270-6474, 1529-2401, doi:10.1523/JNEUROSCI.5088-12.2013, URL http://www.jneurosci.org/content/33/ $40 / 15915$.

[31] (•) F. Zenke, G. Hennequin, W. Gerstner, Synaptic Plasticity in Neural Networks Needs Homeostasis with a Fast Rate Detector, PLoS Comput Biol 9 (11) (2013) e1003330, doi:10.1371/journal.pcbi. 1003330, URL http://dx.doi.org/10.1371/journal.pcbi.1003330.

Mathematical analysis of network dynamics in rate neurons indicating the need for RCP implemented through either weight normalization or metaplastic sliding threshold. 
[32] (•) T. Toyoizumi, M. Kaneko, M. P. Stryker, K. D. Miller, Modeling the Dynamic Interaction of Hebbian and Homeostatic Plasticity, Neuron 84 (2) (2014) 497-510, ISSN 0896-6273, doi:10.1016/j. neuron.2014.09.036, URL http://www.cell.com/article/S0896627314008940/abstract.

The authors show that the time course drop of synaptic strength observed in ocular deprivation experiments cannot be captured by a standard BCM model. Instead the authors suggest a model in which plasticity is intrinsically bistable whereas homeostasis is multiplicative.

[33] P. Yger, M. Gilson, Models of Metaplasticity: A Review of Concepts, Front. Comput. Neurosci (2015) 138doi:10.3389/fncom.2015.00138, URL http://journal.frontiersin.org/article/ $10.3389 /$ fncom. $2015.00138 /$ full.

[34] F. Zenke, W. Gerstner, Hebbian plasticity requires compensatory processes on multiple timescales, Phil. Trans. R. Soc. B 372 (1715) (2017) 20160259, ISSN 0962-8436, 1471-2970, doi:10.1098/rstb.2016.0259, URL http://rstb.royalsocietypublishing.org/content/372/1715/20160259.

[35] W. Senn, H. Markram, M. Tsodyks, An Algorithm for Modifying Neurotransmitter Release Probability Based on Pre- and Postsynaptic Spike Timing, Neural Comput 13 (1) (2001) 35-67, ISSN 0899-7667, doi:10.1162/089976601300014628, URL http://dx.doi.org/10.1162/089976601300014628.

[36] J.-P. Pfister, W. Gerstner, Triplets of Spikes in a Model of Spike Timing-Dependent Plasticity, J Neurosci 26 (38) (2006) 9673-9682, ISSN 0270-6474, 1529-2401, doi:10.1523/JNEUROSCI.1425-06. 2006, URL http://www.jneurosci.org/content/26/38/9673.

[37] (•) C. Clopath, L. Büsing, E. Vasilaki, W. Gerstner, Connectivity reflects coding: a model of voltagebased STDP with homeostasis, Nat Neurosci 13 (3) (2010) 344-352, ISSN 1097-6256, doi:10.1038/nn. 2479, URL http://www .nature.com/neuro/journal/v13/n3/full/nn.2479.html.

Voltage-based plasticity model with parameters extracted from experimental data (see also [113]).

[38] M. Graupner, N. Brunel, Calcium-based plasticity model explains sensitivity of synaptic changes to spike pattern, rate, and dendritic location, Proc Natl Acad Sci U S A 109 (10) (2012) 3991-3996, ISSN 0027-8424, 1091-6490, doi:10.1073/pnas.1109359109, URL http://www.pnas.org/content/109/10/ 3991.

[39] S. Fusi, P. J. Drew, L. F. Abbott, Cascade models of synaptically stored memories., Neuron 45 (4) (2005) 599-611, ISSN 0896-6273, doi:10.1016/j.neuron.2005.02.001, URL http://www.ncbi.nlm.nih . gov/pubmed/15721245.

[40] S. Lahiri, S. Ganguli, A memory frontier for complex synapses, in: Advances in Neural Information Processing Systems, vol. 26, Curran Associates, Inc., Tahoe, USA, 1034-1042, URL http://papers . nips.cc/paper/4872-a-memory-frontier-for-complex-synapses, 2013. 
[41] M. K. Benna, S. Fusi, Computational principles of synaptic memory consolidation, Nat Neurosci advance online publication, ISSN 1097-6256, doi:10.1038/nn.4401, URL http://www.nature.com/ neuro/journal/vaop/ncurrent/full/nn.4401.html.

[42] C. Clopath, L. Ziegler, E. Vasilaki, L. Büsing, W. Gerstner, Tag-Trigger-Consolidation: A Model of Early and Late Long-Term-Potentiation and Depression, PLoS Comput Biol 4 (12) (2008) e1000248, doi:10.1371/journal.pcbi.1000248, URL http://dx.doi.org/10.1371/journal.pcbi.1000248.

[43] A. B. Barrett, G. O. Billings, R. G. M. Morris, M. C. W. van Rossum, State Based Model of LongTerm Potentiation and Synaptic Tagging and Capture, PLoS Comput Biol 5 (1) (2009) e1000259, doi:10.1371/journal.pcbi.1000259, URL http://dx.doi.org/10.1371/journal.pcbi.1000259.

[44] L. Ziegler, F. Zenke, D. B. Kastner, W. Gerstner, Synaptic Consolidation: From Synapses to Behavioral Modeling, J Neurosci 35 (3) (2015) 1319-1334, ISSN 0270-6474, 1529-2401, doi:10.1523/JNEUROSCI. 3989-14.2015, URL http://www. jneurosci.org/content/35/3/1319.

[45] C. D. Rittenhouse, H. Z. Shouval, M. A. Paradiso, M. F. Bear, Monocular deprivation induces homosynaptic long-term depression in visual cortex, Nature 397 (6717) (1999) 347-350, ISSN 0028-0836, doi: 10.1038/16922, URL http://www.nature.com/nature/journal/v397/n6717/abs/397347a0.html.

[46] T. Rose, J. Jaepel, M. Hübener, T. Bonhoeffer, Cell-specific restoration of stimulus preference after monocular deprivation in the visual cortex, Science 352 (6291) (2016) 1319-1322, ISSN 00368075, 1095-9203, doi:10.1126/science.aad3358, URL http://science.sciencemag.org/content/ $352 / 6291 / 1319$.

[47] K. Nakazawa, L. D. Sun, M. C. Quirk, L. Rondi-Reig, M. A. Wilson, S. Tonegawa, Hippocampal CA3 NMDA Receptors Are Crucial for Memory Acquisition of One-Time Experience, Neuron 38 (2) (2003) 305-315, ISSN 0896-6273, doi:10.1016/S0896-6273(03)00165-X, URL http://www.sciencedirect. com/science/article/pii/S089662730300165X.

[48] I. Lee, R. P. Kesner, Differential contributions of dorsal hippocampal subregions to memory acquisition and retrieval in contextual fear-conditioning, Hippocampus 14 (3) (2004) 301-310, ISSN 1098-1063, doi: 10.1002/hipo.10177, URL http://onlinelibrary.wiley.com/doi/10.1002/hipo.10177/abstract.

[49] A. Lazar, G. Pipa, J. Triesch, SORN: A Self-Organizing Recurrent Neural Network, Front Comput Neurosci 3, ISSN 1662-5188, doi:10.3389/neuro.10.023.2009, URL http://www.ncbi.nlm.nih.gov/ pmc/articles/PMC2773171/.

[50] (•) A. Litwin-Kumar, B. Doiron, Formation and maintenance of neuronal assemblies through synaptic 
plasticity, Nat Commun 5, doi:10.1038/ncomms6319, URL http://www.nature.com/ncomms/2014/ 141114/ncomms6319/full/ncomms6319.html.

This article demonstrates stable learning and transient recall $(\sim 100 \mathrm{~ms})$ of Hebbian cell assemblies in a recurrent network model through the combination of a plausible model of STDP with two homeostatic processes: a fast one that is implemented as a renormalization of the sum of all synaptic weights onto a given neuron every $20 \mathrm{~ms}$, plus a rapid firing rate homeostasis (FRH) implemented by plasticity ISP following a model by [86] (see also [82]).

[51] S. El Boustani, P. Yger, Y. Frégnac, A. Destexhe, Stable Learning in Stochastic Network States, J Neurosci 32 (1) (2012) 194 -214, doi:10.1523/JNEUROSCI.2496-11.2012, URL http://www. jneurosci. org/content/32/1/194. abstract.

[52] J. Gjorgjieva, C. Clopath, J. Audet, J.-P. Pfister, A triplet spike-timing-dependent plasticity model generalizes the Bienenstock-Cooper-Munro rule to higher-order spatiotemporal correlations, Proc Natl Acad Sci U S A 108 (48) (2011) 19383 -19388, doi:10.1073/pnas.1105933108, URL http://www. pnas . org/content/108/48/19383. abstract.

[53] M. C. W. van Rossum, G. Q. Bi, G. G. Turrigiano, Stable Hebbian Learning from Spike TimingDependent Plasticity, J Neurosci 20 (23) (2000) 8812-8821, ISSN 0270-6474, 1529-2401, URL http: //www.jneurosci.org/content/20/23/8812.

[54] Y. Y. Huang, A. Colino, D. K. Selig, R. C. Malenka, The influence of prior synaptic activity on the induction of long-term potentiation, Science 255 (5045) (1992) 730-733, ISSN 0036-8075.

[55] J. Aoto, C. I. Nam, M. M. Poon, P. Ting, L. Chen, Synaptic Signaling by All-Trans Retinoic Acid in Homeostatic Synaptic Plasticity, Neuron 60 (2) (2008) 308-320, ISSN 0896-6273, doi:10.1016/j.neuron. 2008.08.012, URL http://www.sciencedirect.com/science/article/pii/S0896627308007071.

[56] K. Ibata, Q. Sun, G. G. Turrigiano, Rapid Synaptic Scaling Induced by Changes in Postsynaptic Firing, Neuron 57 (6) (2008) 819-826, ISSN 0896-6273, doi:10.1016/j.neuron.2008.02.031, URL http: //www.sciencedirect.com/science/article/pii/S0896627308002134.

[57] S. D. Greenhill, A. Ranson, K. Fox, Hebbian and Homeostatic Plasticity Mechanisms in Regular Spiking and Intrinsic Bursting Cells of Cortical Layer 5, Neuron 88 (3) (2015) 539-552, ISSN 08966273, doi:10.1016/j.neuron.2015.09.025, URL http://www.sciencedirect.com/science/article/ $\mathrm{pii} / \mathrm{S} 0896627315008144$.

[58] B. R. Christie, W. C. Abraham, Priming of associative long-term depression in the dentate gyrus by theta frequency synaptic activity, Neuron 9 (1) (1992) 79-84, ISSN 0896-6273, 
doi:10.1016/0896-6273(92)90222-Y, URL http://www.sciencedirect.com/science/article/pii/ $089662739290222 \mathrm{Y}$.

[59] A. Goel, B. Jiang, L. W. Xu, L. Song, A. Kirkwood, H.-K. Lee, Cross-modal regulation of synaptic AMPA receptors in primary sensory cortices by visual experience, Nat Neurosci 9 (8) (2006) 10011003, ISSN 1097-6256, doi:10.1038/nn1725, URL http://www.nature.com/neuro/journal/v9/n8/ abs/nn1725.html.

[60] M. Kaneko, D. Stellwagen, R. C. Malenka, M. P. Stryker, Tumor Necrosis Factor- $\alpha$ Mediates One Component of Competitive, Experience-Dependent Plasticity in Developing Visual Cortex, Neuron 58 (5) (2008) 673-680, ISSN 0896-6273, doi:10.1016/j.neuron.2008.04.023, URL http://www. sciencedirect.com/science/article/pii/S0896627308003772.

[61] B. Mockett, C. Coussens, W. C. Abraham, NMDA receptor-mediated metaplasticity during the induction of long-term depression by low-frequency stimulation, Eur J Neurosci 15 (11) (2002) 18191826, ISSN 1460-9568, doi:10.1046/j.1460-9568.2002.02008.x, URL http://onlinelibrary.wiley. com/doi/10.1046/j.1460-9568.2002.02008.x/abstract.

[62] L. Li, M. A. Gainey, J. E. Goldbeck, D. E. Feldman, Rapid homeostasis by disinhibition during whisker map plasticity, PNAS 111 (4) (2014) 1616-1621, ISSN 0027-8424, 1091-6490, doi:10.1073/ pnas.1312455111, URL http://www.pnas.org/content/111/4/1616.

[63] L. Benuskova, W. Abraham, STDP rule endowed with the BCM sliding threshold accounts for hippocampal heterosynaptic plasticity, J Comput Neurosci 22 (2) (2007) 129-133, ISSN 0929-5313, doi:10.1007/s10827-006-0002-x, URL http://www.springerlink.com/content/b51224m81192h232/ abstract/.

[64] T. O'Leary, D. J. A. Wyllie, Neuronal homeostasis: time for a change?, The Journal of Physiology 589 (20) (2011) 4811-4826, ISSN 1469-7793, doi:10.1113/jphysiol.2011.210179, URL http: //onlinelibrary.wiley.com/doi/10.1113/jphysiol.2011.210179/abstract.

[65] A. Williams, T. O’Leary, E. Marder, Homeostatic Regulation of Neuronal Excitability, Scholarpedia 8 (1) (2013) 1656, ISSN 1941-6016, doi:10.4249/scholarpedia.1656, URL http://www .scholarpedia. org/article/Homeostatic_Regulation_of_Neuronal_Excitability.

[66] K. J. Aström, R. M. Murray, Feedback Systems: An Introduction for Scientists and Engineers, Princeton University Press, ISBN 978-1-4008-2873-9, google-Books-ID: cdG9fNqTDS8C, 2010.

[67] L. C. Udeigwe, P. W. Munro, G. B. Ermentrout, Emergent Dynamical Properties of the BCM Learning 
Rule, J. Math. Neurosc. 7 (1) (2017) 2, ISSN 2190-8567, doi:10.1186/s13408-017-0044-6, URL https: //link.springer.com/article/10.1186/s13408-017-0044-6.

[68] P. Yger, K. D. Harris, The Convallis Rule for Unsupervised Learning in Cortical Networks, PLoS Comput Biol 9 (10) (2013) e1003272, doi:10.1371/journal.pcbi.1003272, URL http://dx.doi.org/ 10.1371/journal.pcbi.1003272.

[69] R. Kempter, W. Gerstner, J. L. van Hemmen, Intrinsic stabilization of output rates by spikebased Hebbian learning, Neural Comput 13 (12) (2001) 2709-2741, ISSN 0899-7667, doi:10.1162/ 089976601317098501 , URL http://www.ncbi.nlm.nih.gov/pubmed/11705408.

[70] W. M. Kistler, J. L. Hemmen, Modeling synaptic plasticity in conjunction with the timing of preand postsynaptic action potentials, Neural Computation 12 (2) (2000) 385-405, URL http://www. mitpressjournals.org/doi/abs/10.1162/089976600300015844.

[71] J. Rubin, D. D. Lee, H. Sompolinsky, Equilibrium Properties of Temporally Asymmetric Hebbian Plasticity, Phys. Rev. Lett. 86 (2) (2001) 364-367, doi:10.1103/PhysRevLett.86.364, URL http:// link.aps.org/doi/10.1103/PhysRevLett.86.364.

[72] R. Gütig, R. Aharonov, S. Rotter, H. Sompolinsky, Learning Input Correlations through Nonlinear Temporally Asymmetric Hebbian Plasticity, J Neurosci 23 (9) (2003) 3697-3714, ISSN 0270-6474, 1529-2401, URL http://www.jneurosci.org/content/23/9/3697.

[73] A. Morrison, A. Aertsen, M. Diesmann, Spike-timing-dependent plasticity in balanced random networks., Neural Comput 19 (6) (2007) 1437-67, ISSN 0899-7667, doi:10.1162/neco.2007.19.6.1437, URL http://www.ncbi.nlm.nih.gov/pubmed/17444756.

[74] M. Gilson, A. N. Burkitt, D. B. Grayden, D. A. Thomas, J. L. Hemmen, Emergence of network structure due to spike-timing-dependent plasticity in recurrent neuronal networks. I. Input selectivity-strengthening correlated input pathways, Biol Cybern 101 (2) (2009) 81-102, ISSN 03401200, 1432-0770, doi:10.1007/s00422-009-0319-4, URL http://www.springerlink.com/content/ 8441p585w93u0576/.

[75] G. Billings, M. C. W. van Rossum, Memory Retention and Spike-Timing-Dependent Plasticity, J Neurophysiol 101 (6) (2009) 2775 -2788, doi:10.1152/jn.91007.2008, URL http://jn.physiology. org/content/101/6/2775. abstract.

[76] S. Kunkel, M. Diesmann, A. Morrison, Limits to the development of feed-forward structures in large recurrent neuronal networks, Front Comput Neurosci 4 (2011) 160, doi:10.3389/fncom.2010. 
00160, URL http://www.frontiersin.org/Computational_Neuroscience/10.3389/fncom.2010. 00160/pdf/abstract.

[77] V. Delattre, D. Keller, M. Perich, H. Markram, E. B. Muller, Network-timing-dependent plasticity, Front. Cell. Neurosci. (2015) 220doi:10.3389/fncel.2015.00220, URL http://journal.frontiersin . org/article/10.3389/fncel.2015.00220/full.

[78] G.-q. Bi, M.-m. Poo, Synaptic modification of correlated activity: Hebb's postulate revisited, Ann. Rev. Neurosci. 24 (2001) 139-166.

[79] H. Markram, W. Gerstner, P. J. Sjöström, A history of spike-timing-dependent plasticity, Front Synaptic Neurosci 3 (2011) 4, doi:10.3389/fnsyn.2011.00004, URL http://www.frontiersin.org/ Synaptic_Neuroscience/10.3389/fnsyn.2011.00004/pdf/abstract.

[80] A. X. Yee, Y.-T. Hsu, L. Chen, A metaplasticity view of the interaction between homeostatic and Hebbian plasticity, Phil. Trans. R. Soc. B 372 (1715) (2017) 20160155, ISSN 0962-8436, 1471-2970, doi:10.1098/rstb.2016.0155, URL http://rstb.royalsocietypublishing.org/content/372/1715/ 20160155.

[81] B. Babadi, L. F. Abbott, Stability and Competition in Multi-spike Models of Spike-Timing Dependent Plasticity, PLOS Comput Biol 12 (3) (2016) e1004750, ISSN 1553-7358, doi:10. 1371/journal.pcbi.1004750, URL http://journals.plos.org/ploscompbiol/article?id=10.1371/ journal.pcbi.1004750.

[82] (•) F. Zenke, E. J. Agnes, W. Gerstner, Diverse synaptic plasticity mechanisms orchestrated to form and retrieve memories in spiking neural networks, Nat Commun 6, doi:doi:10.1038/ncomms7922, URL http://dx.doi.org/10.1038/ncomms7922.

Here the authors demonstrate stable learning and recall of Hebbian cell assemblies in a recurrent network model by combining a plausible STDP model with heterosynaptic and transmitter-induced plasticity which both act as RCPs to prevents runaway activity (see also [50]).

[83] J. Y. Delgado, J. F. Gomez-Gonzalez, N. S. Desai, Pyramidal Neuron Conductance State Gates Spike-Timing-Dependent Plasticity, J Neurosci 30 (47) (2010) 15713-15725, ISSN 0270-6474, doi:10. 1523/JNEUROSCI.3068-10.2010, URL http://www.jneurosci.org/cgi/doi/10.1523/JNEUROSCI . $3068-10.2010$.

[84] S. Lim, J. L. McKee, L. Woloszyn, Y. Amit, D. J. Freedman, D. L. Sheinberg, N. Brunel, Inferring learning rules from distributions of firing rates in cortical neurons, Nat Neurosci advance online publication, ISSN 1097-6256, doi:10.1038/nn.4158, URL http://www.nature.com/neuro/journal/vaop/ ncurrent/full/nn.4158.html. 
[85] K. A. Wilmes, H. Sprekeler, S. Schreiber, Inhibition as a Binary Switch for Excitatory Plasticity in Pyramidal Neurons, PLOS Comput Biol 12 (3) (2016) e1004768, ISSN 1553-7358, doi:10. 1371/journal.pcbi.1004768, URL http://journals.plos.org/ploscompbiol/article?id=10.1371/ journal.pcbi.1004768.

[86] T. P. Vogels, H. Sprekeler, F. Zenke, C. Clopath, W. Gerstner, Inhibitory Plasticity Balances Excitation and Inhibition in Sensory Pathways and Memory Networks, Science 334 (6062) (2011) 1569-1573, doi: 10.1126/science.1211095, URL http://www.sciencemag.org/content/334/6062/1569. abstract.

[87] Y. Luz, M. Shamir, Balancing Feed-Forward Excitation and Inhibition via Hebbian Inhibitory Synaptic Plasticity, PLoS Comput Biol 8 (1) (2012) e1002334, doi:10.1371/journal.pcbi.1002334, URL http: //dx.doi.org/10.1371/journal.pcbi.1002334.

[88] C. Clopath, T. P. Vogels, R. C. Froemke, H. Sprekeler, Receptive field formation by interacting excitatory and inhibitory synaptic plasticity, bioRxiv (2016) 066589doi:10.1101/066589, URL http://www . biorxiv. org/content/early/2016/07/29/066589.

[89] L. Wang, A. Maffei, Inhibitory Plasticity Dictates the Sign of Plasticity at Excitatory Synapses, J. Neurosci. 34 (4) (2014) 1083-1093, ISSN 0270-6474, 1529-2401, doi:10.1523/JNEUROSCI.4711-13. 2014, URL http://www.jneurosci.org/content/34/4/1083.

[90] M. F. Bear, W. Singer, Modulation of visual cortical plasticity by acetylcholine and noradrenaline, Nature 320 (1986) 172-176.

[91] U. Frey, R. G. M. Morris, Synaptic tagging: implications for late maintenance of hippocampal long-term potentiation, Trends Neurosci 21 (5) (1998) 181-188, ISSN 0166-2236, doi:10.1016/S0166-2236(97) 01189-2, URL http://www.sciencedirect.com/science/article/pii/S0166223697011892.

[92] J. C. Zhang, P. M. Lau, G. Q. Bi, Gain in sensitivity and loss in temporal contrast of STDP by dopaminergic modulation at hippocampal synapses., Proc. Natl. Aca. Sci. USA 106 (2009) 1302813033.

[93] V. Pawlak, J. R. Wickens, A. Kirkwood, J. N. D. Kerr, Timing is not Everything: Neuromodulation Opens the STDP Gate, Front Synaptic Neurosci 2 (2010) 146, ISSN 1663-3563, doi:10.3389/fnsyn. 2010.00146, URL http://www.ncbi.nlm.nih.gov/pubmed/21423532.

[94] N. Frémaux, W. Gerstner, Neuromodulated Spike-Timing-Dependent Plasticity, and Theory of ThreeFactor Learning Rules, Front Neural Circuits (2016) 85doi:10.3389/fncir.2015.00085, URL http:// journal.frontiersin.org/article/10.3389/fncir.2015.00085/full. 
[95] N. Hardingham, J. Dachtler, K. Fox, The role of nitric oxide in pre-synaptic plasticity and homeostasis, Front Cell Neurosci 7 (2013) 190, doi:10.3389/fncel.2013.00190.

[96] F. Crespi, Z. L. Rossetti, Pulse of Nitric Oxide Release in Response to Activation of N-Methyl-daspartate Receptors in the Rat Striatum: Rapid Desensitization, Inhibition by Receptor Antagonists, and Potentiation by Glycine, J Pharmacol Exp Ther 309 (2) (2004) 462-468, ISSN 0022-3565, 15210103, doi:10.1124/jpet.103.061069, URL http://jpet.aspetjournals.org/content/309/2/462.

[97] Y. Sweeney, J. Hellgren Kotaleski, M. H. Hennig, A Diffusive Homeostatic Signal Maintains Neural Heterogeneity and Responsiveness in Cortical Networks, PLoS Comput Biol 11 (7) (2015) e1004389, doi:10.1371/journal.pcbi.1004389, URL http://dx.doi.org/10.1371/journal.pcbi.1004389.

[98] W. Schultz, Multiple dopamine functions at different time courses, Annu. Rev. Neurosci. 30 (2007) 259-288.

[99] Z. Gu, Z. Yan, Bidirectional Regulation of Ca2+/Calmodulin-Dependent Protein Kinase II Activity by Dopamine D4 Receptors in Prefrontal Cortex, Mol Pharmacol 66 (4) (2004) 948-955, ISSN 0026-895X, 1521-0111, doi:10.1124/mol.104.001404, URL http://molpharm.aspetjournals.org/content/66/ $4 / 948$.

[100] R. L. Redondo, R. G. M. Morris, Making memories last: the synaptic tagging and capture hypothesis, Nat Rev Neurosci 12 (1) (2011) 17-30, ISSN 1471-003X, doi:10.1038/nrn2963, URL http://www . nature.com/nrn/journal/v12/n1/abs/nrn2963.html.

[101] J. Lisman, Glutamatergic synapses are structurally and biochemically complex because of multiple plasticity processes: long-term potentiation, long-term depression, short-term potentiation and scaling, Phil. Trans. R. Soc. B 372 (1715) (2017) 20160260, ISSN 0962-8436, 1471-2970, doi:10.1098/rstb.2016. 0260, URL http://rstb.royalsocietypublishing.org/content/372/1715/20160260.

[102] R. Min, T. Nevian, Astrocyte signaling controls spike timing-dependent depression at neocortical synapses, Nat Neurosci 15 (5) (2012) 746-753, ISSN 1097-6256, doi:10.1038/nn.3075, URL http: //www. nature.com/neuro/journal/v15/n5/full/nn.3075.html.

[103] A. Volterra, N. Liaudet, I. Savtchouk, Astrocyte Ca2+ signalling: an unexpected complexity, Nat Rev Neurosci 15 (5) (2014) 327-335, ISSN 1471-003X, doi:10.1038/nrn3725, URL http://www.nature. com/nrn/journal/v15/n5/abs/nrn3725.html.

[104] M. De Pittà, N. Brunel, A. Volterra, Astrocytes: Orchestrating synaptic plasticity?, Neuroscience 323 (2016) 43-61, ISSN 0306-4522, doi:10.1016/j.neuroscience.2015.04.001, URL http://www . sciencedirect.com/science/article/pii/S0306452215003188. 
[105] G. S. Lynch, T. Dunwiddie, V. Gribkoff, Heterosynaptic depression: a postsynaptic correlate of long-term potentiation, Nature 266 (5604) (1977) 737-739, doi:10.1038/266737a0, URL http://www . nature.com/nature/journal/v266/n5604/abs/266737a0.html.

[106] S. Royer, D. Paré, Conservation of total synaptic weight through balanced synaptic depression and potentiation, Nature 422 (6931) (2003) 518-522, ISSN 0028-0836, doi:10.1038/nature01530, URL http: //www.nature.com/nature/journal/v422/n6931/full/nature01530.html.

[107] (•) M. Chistiakova, N. M. Bannon, M. Bazhenov, M. Volgushev, Heterosynaptic Plasticity Multiple Mechanisms and Multiple Roles, Neuroscientist 20 (5) (2014) 483-498, ISSN 1073-8584, 1089-4098, doi:10.1177/1073858414529829, URL http://nro.sagepub.com/content/20/5/483.

A comprehensive review of heterosynaptic plasticity in experiments and its putative role as a RCP.

[108] (•) W. Oh, L. Parajuli, K. Zito, Heterosynaptic Structural Plasticity on Local Dendritic Segments of Hippocampal CA1 Neurons, Cell Reports 10 (2) (2015) 162-169, ISSN 2211-1247, doi:10.1016/j.celrep. 2014.12.016, URL http://www.sciencedirect.com/science/article/pii/S2211124714010456.

This article shows that when a cluster of spines is stimulated through glutamate uncaging, which under control conditions leads to structural growth which is presumably related to Hebbian plasticity, nearby unstimulated spines shrink which corresponds to heterosynaptic depression.

[109] (•) M. Volgushev, J.-Y. Chen, V. Ilin, R. Goz, M. Chistiakova, M. Bazhenov, Partial Breakdown of Input Specificity of STDP at Individual Synapses Promotes New Learning, J. Neurosci. 36 (34) (2016) 8842-8855, ISSN 0270-6474, 1529-2401, doi:10.1523/JNEUROSCI.0552-16.2016, URL http: //www. jneurosci.org/content/36/34/8842.

A recent experimental study with additional modelling demonstrating that heterosynaptic plasticity co-occurs with input-specific homosynaptic plasticity and can effectively stabilize runaway LTP.

[110] Q. Zhou, H. W. Tao, M.-M. Poo, Reversal and Stabilization of Synaptic Modifications in a Developing Visual System, Science 300 (5627) (2003) 1953-1957, doi:10.1126/science.1082212, URL http://www . sciencemag.org/cgi/content/abstract/sci;300/5627/1953.

[111] J. N. Bourne, K. M. Harris, Coordination of size and number of excitatory and inhibitory synapses results in a balanced structural plasticity along mature hippocampal CA1 dendrites during LTP, Hippocampus 21 (4) (2011) 354-373, ISSN 1098-1063, doi:10.1002/hipo.20768, URL http: //onlinelibrary.wiley.com/doi/10.1002/hipo.20768/abstract.

[112] W. C. Abraham, B. Logan, A. Wolff, L. Benuskova, "Heterosynaptic" LTD in the Dentate Gyrus of Anesthetized Rat Requires Homosynaptic Activity, J Neurophysiol 98 (2) (2007) 1048-1051, ISSN 00223077, 1522-1598, doi:10.1152/jn.00250.2007, URL http://jn.physiology.org/content/98/2/1048. 
[113] (•) P. Jedlicka, L. Benuskova, W. C. Abraham, A Voltage-Based STDP Rule Combined with Fast BCM-Like Metaplasticity Accounts for LTP and Concurrent "Heterosynaptic" LTD in the Dentate Gyrus In Vivo, PLoS Comput Biol 11 (11) (2015) e1004588, doi:10.1371/journal.pcbi.1004588, URL http://dx.doi.org/10.1371/journal.pcbi.1004588.

A biophysical plasticity model is presented and fitted to experimental data. Interestingly, best fits are obtained for parameters which are suggestive of a rapid $(\sim 10$ s) sliding threshold mechanism reminiscent of the BCM model.

[114] G. Daoudal, D. Debanne, Long-Term Plasticity of Intrinsic Excitability: Learning Rules and Mechanisms, Learn Mem 10 (6) (2003) 456-465, ISSN 1072-0502, 1549-5485, doi:10.1101/lm.64103, URL http://learnmem.cshlp.org/content/10/6/456.

[115] P. Sjöström, E. Rancz, A. Roth, M. Häusser, Dendritic excitability and synaptic plasticity, Physiol Rev 88 (2) (2008) 769, ISSN 0031-9333, doi:10.1152/physrev.00016.2007., URL http://scholar.google.com/scholar?hl=en\&btnG=Search\&q=intitle: Dendritic+ Excitability+and+Synaptic+Plasticity\#0.

[116] D. Johnston, R. Narayanan, Active dendrites: colorful wings of the mysterious butterflies, Trends in Neurosciences 31 (6) (2008) 309-316, ISSN 0166-2236, doi:10.1016/j.tins.2008.03.004, URL http: //www.sciencedirect.com/science/article/pii/S0166223608001197.

[117] T. O'Leary, A. Williams, A. Franci, E. Marder, Cell Types, Network Homeostasis, and Pathological Compensation from a Biologically Plausible Ion Channel Expression Model, Neuron 82 (4) (2014) 809-821, ISSN 0896-6273, doi:10.1016/j.neuron.2014.04.002, URL http://www.sciencedirect.com/ science/article/pii/S089662731400292X. 
Figure 1: Comparison of the typical timescale of different forms of homeostatic plasticity in models and in experiments. (a) Weight normalization in models. Here we plot the characteristic timescale on which synaptic weights are either normalized or scaled. (b) Synaptic scaling in experiments. Here we plot the typical time at which synaptic scaling is observed. (c) Models of metaplasticity. We plot the characteristic timescale on which the learning rule changes. (d) Metaplasticity in experiments. Here we show the typical timescale at which metaplasticity is observed.

Figure 2: A control theoretic view of homeostatic plasticity and Hebbian learning. (a) The coupled dynamics of Hebbian plasticity and neural activity constitutes an unstable dynamical system. Homeostatic plasticity, and more generally any compensatory mechanism, can be viewed as a controller that observes aspects of neuronal activity and synaptic strengths, and uses these observations to compute a feedback control signal that acts on synaptic dynamics so as to stabilize circuit properties. (b) The cart pole problem is a simple example of stabilizing a non-linear dynamical system with feedback. The task of the controller is to exert horizontal forces on the cart to maintain the rod $(m=1 \mathrm{~kg})$ in an upright position. For simplicity we assume a weightless cart with no spatial constraints on the length of the track. The controller has access to an exponential average (time constant $\tau$ ) over recent observations of both the angle $\theta$ and angular velocity $\dot{\theta}$ of the pole. For small values of $\tau$ the controller can successfully maintain the rod upright $(\theta \approx 0$; top panel). However, as $\tau$ and the associated time lag in the observed quantities gets larger, oscillations arise (middle panel) and eventually the system becomes unstable (bottom panel). 
Figure 3: Instability in different plasticity models. (a) Unstable oscillations in the BCM model for a simple feed-forward circuit [6, 11]. Model: $\tau_{w} \frac{d w}{d t}=\alpha \nu_{\text {in }} \nu_{\text {out }}\left(\nu_{\text {out }}-\beta \bar{\nu}_{\text {out }}^{2}\right), \tau_{\mathrm{c}} \frac{d}{d t} \bar{\nu}_{\text {out }}=\nu_{\text {out }}-\bar{\nu}_{\text {out }}$ with $\nu_{\text {out }}=w \nu_{\text {in }}, \tau_{w}=0.9 \tau_{\mathrm{c}}, \nu_{\text {in }} \equiv 1$ in arbitrary units (a.u.) and $\alpha$ and $\beta$ are dimensionful scalar constants that ensure correct units; we take simply $\alpha=\beta=1$. Here $\bar{\nu}_{\text {out }}$ can be thought of as the observation of a controller, corresponding to an average of output neuronal activity $\nu_{\text {out }}$ over time-scale $\tau_{\mathrm{c}}$. Moreover, the multiplicative term in parenthesis in the weight dynamics can be interpreted as a control signal that modulates both the sign and amplitude of associative plasticity, dictating a stabilizing anti-Hebbian rule if the recent average $\bar{\nu}_{\text {out }}$ is too large. If the control dynamics $\tau_{\mathrm{c}}$ is too slow relative to the synaptic plasticity dynamics $\tau_{w}$, unstable oscillations arise. (b-d) Runaway activity in a recurrent neural network simulation consisting of 25000 excitatory and inhibitory integrate-and-fire neurons and plastic excitatory synapses using a minimal triplet STDP model [36] with different homeostatic mechanisms such as sliding threshold metaplasticity (violet) and synaptic scaling (green), both described in detail in [31]. (b) Population firing rate as a function of time. (c,d) Raster plot of spiking activity. The bottom 3 groups of 100 neurons received rate modulated spiking input with $100 \mathrm{~ms}$ correlation time constant to emulate sensory input to a small set of neurons. The timescale of sliding threshold metaplasticity was $\tau_{\mathrm{c}}=3$ mins. The timescale for the rate detector and the scaling dynamics for synaptic scaling were $\tau_{\mathrm{c}}=10 \mathrm{~s}$ and $\tau_{\mathrm{scl}}=1 \mathrm{~h}$ respectively. Because of these slow stabilization dynamics, the fast interplay between Hebbian plasticity and recurrent network dynamics leads to rapid population firing rate destabilization within 10 to 20 seconds for both learning rules.

Figure 4: (a) Schematic representation of the many potential loci of rapid compensatory processes. (b) Example of a plastic network model displaying attractor dynamics in three distinct cell assemblies. Because these dynamics outlast the stimulus that triggers them they have been considered as neural substrate for working memory. The top panel shows a spike raster, whereas the bottom panel shows the population firing rates of the three cell assemblies. This particular model uses an augmented triplet STDP learning rule (cf. Fig. 3) in which heterosynaptic plasticity and single-synapse-level plasticity have been added as stabilizing RCPs. Moreover, this model relies on a slow form of inhibitory plasticity which normalizes the overall network activity and ensures that cell assemblies do not grow indefinitely over time by recruiting additional neurons. This provides a proof of principle that a biologically inspired learning rule can indeed be stabilized by a sensible combination of RCPs, whereas the same learning rule endowed with slow compensatory mechanisms leads to run-away dynamics (cf. Fig. 3b-d). Figure adapted from Zenke et al. [82]. 
a

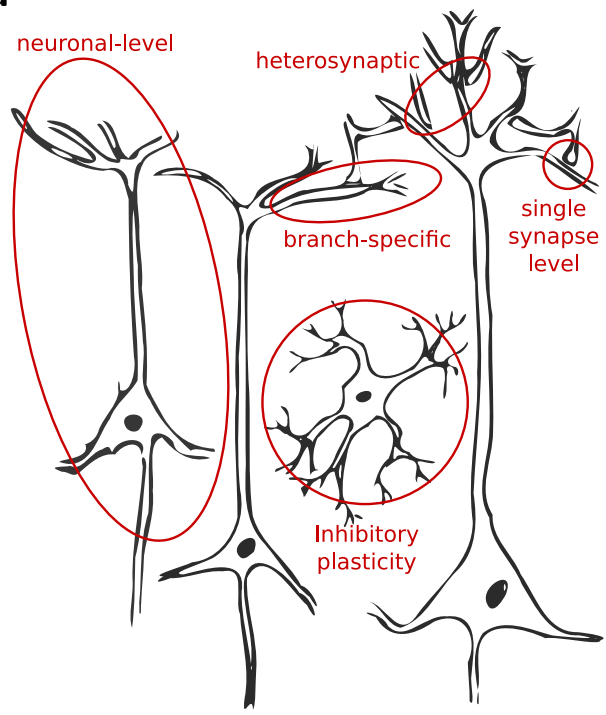

b

Stimulation times: "

Spikes:

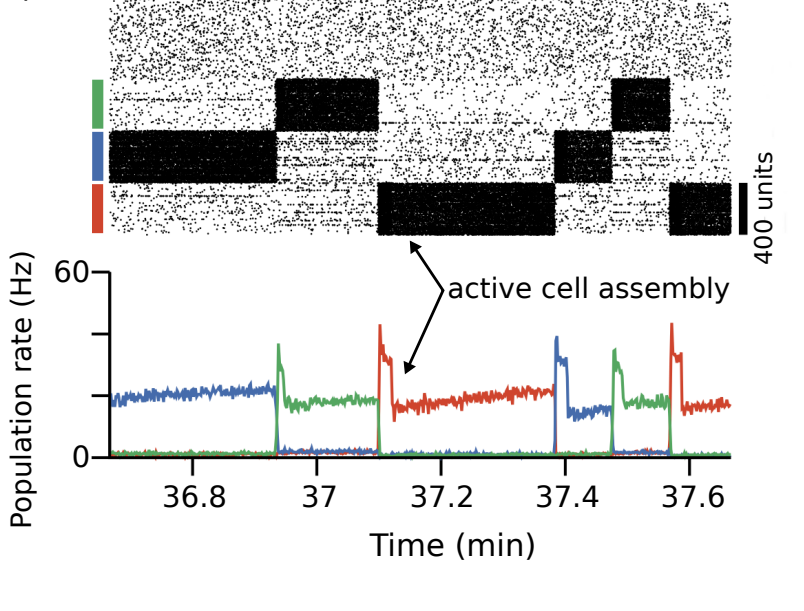




\section{a}

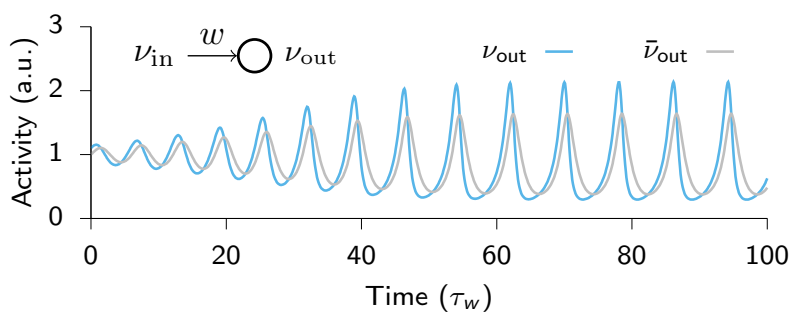

b
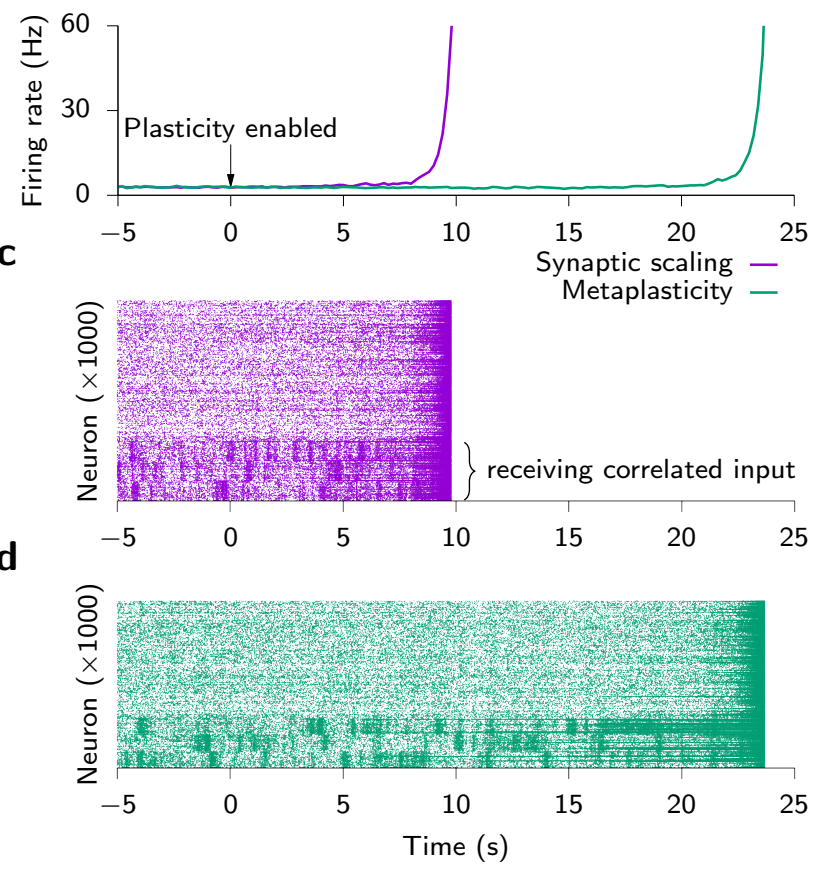


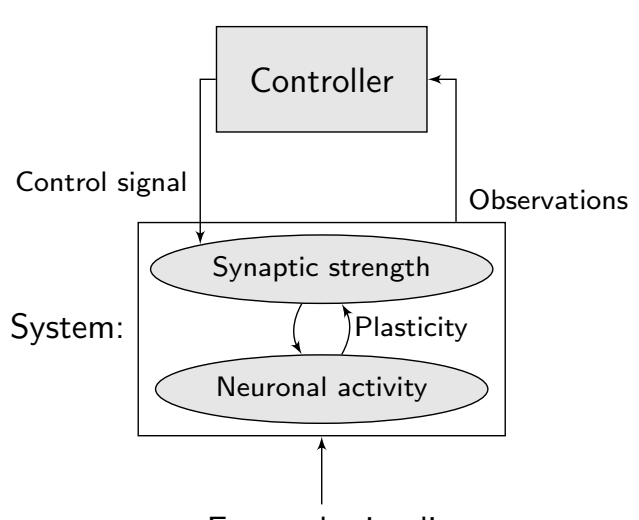

External stimuli
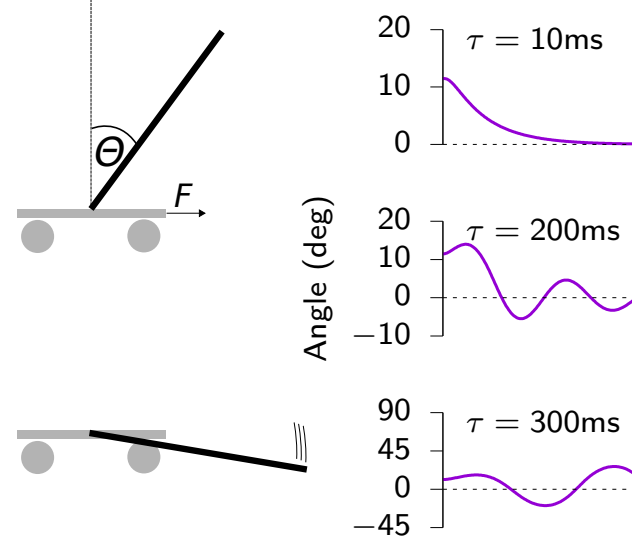
a

week
day
hour
minute
second

C hour
minute
second
Weight normalization in models

Toyoizumi et al. [32]

van Rossum et al. [53], Zenke et al. [31] *

Litwin-Kumar and Doiron [50] *

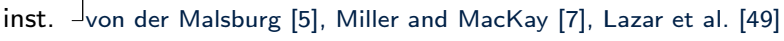

Models of metaplasticity

Pfister and Gerstner [36]

Zenke et al. [31] *

Benuskova and Abraham [63]

Jedlicka et al. [113]

Gjorgjieva et al. [52]

El Boustani et al. [51] *

Clopath et al. [37] *

* network simulations
Synaptic scaling in experiments

Goel et al. [59]
Kaneko et al. [60]

$\mathrm{Li}$ et al. [62]

Turrigiano et al. [13]

day

Greenhill et al. [57]

Aoto et al. [55], Ibata et al. [56]

Metaplasticity in experiments

Christie and Abraham [58]

Huang et al. [54]

$10 \mathrm{~min}$

Mockett et al. [61] 